\title{
Quitlines in North America: Evidence Base and Applications
}

DEBORAH J. OSSIP-KLEIN, PHD; SCOTT MCINTOSH, PHD

\begin{abstract}
Quitlines provide a model for the translation of research findings to public health application. Quitlines are currently in operation in more than half of US states, in Canada, and in multiple countries globally. Overall, when implemented correctly, quitlines have been shown to be efficacious and effective. Multiple quitline models are in use, but there is no evidence on the relative effectiveness of one over the other. Differences have been demonstrated for the efficacy of quit-
\end{abstract}

$\mathbf{Q}$ uitlines provide an exemplary model of how research findings can be translated into public health application. The field began with a handful of researchers in the early $1980 \mathrm{~s}$ and experienced marked growth through the past 2 decades. Currently, in North America, quitlines are in operation in more than 30 US states, a number of major American health systems, and multiple Canadian provinces. Quitlines are also available in multiple countries globally, including Denmark, France, Germany, Iceland, Ireland, Italy, Netherlands, Norway, Poland, Portugal, Spain, Sweden, Switzerland, the United Kingdom, Australia, New Zealand, and others.

Overall, when implemented correctly, quitlines have been shown to be both efficacious and effective. The beneficial impact of quitlines has been supported by 3 meta-analyses ${ }^{1-3}$ and by multiple individual studies.

The purpose of this article is to provide an overview of the goals, range of services, and models for quitlines; to summarize the evidence base for what works; to discuss a special and growing application of quitlines to adolescents; and to provide a look at directions for the field.

From the University of Rochester School of Medicine and Dentistry and the James P. Wilmot Cancer Center, Rochester, New York.

Preparation of this manuscript was supported in part by $\mathrm{Na}$ tional Cancer Institute Grants R01-CA80283 and R01-CA67594 (to DJO-K).

Correspondence: Deborah J. Ossip-Klein, Ph.D., James P. Wilmot Cancer Center, University of Rochester School of Medicine, 601 Elmwood Avenue, Box 704, Rochester, NY 14642 (E-mail: deborah_ossipklein@urmc.rochester.edu). lines for specific applications, with the strongest evidence base for application as a primary intervention or as follow-up for hospitalized patients and particularly for cardiac patients. The evidence base for both reactive and proactive services is reviewed, and future directions to continue to advance the field are discussed. KEY INDEXING TERMS: Smoking; Telephone; Telecounseling; Quitline. [Am J Med Sci 2003;326(4):201-205.]

\section{Overview: Goals, Range of Services, Quitline Models}

Goals. Quitlines can have the greatest potential impact when used as part of a comprehensive tobacco control effort. Within this context, quitlines can have 2 primary goals to maximize their public health influence. First, quitlines can provide direct service to help smokers quit. The Centers for Disease Control (CDC) estimates that only $2.5 \%$ of smokers quit permanently each year ${ }^{4}$; similar figures have also been reported for the United Kingdom. ${ }^{5}$ These rates suggest that there is considerable opportunity for quitlines to directly affect smoking cessation behavior; even a small percentage increase in quit rates has the potential to translate into meaningful public health change.

A second primary goal of quitlines is to increase the number of smokers making a quit attempt each year, by awareness raising generated from promotional campaigns linked with quitlines. Even if success rates for quitting remain constant among those who try, increasing the number of smokers who make a quit attempt can yield a greater number of total quitters. About half of smokers do not attempt to quit in a given year, again indicating an opportunity for intervention.

A third related goal is to provide access to information and counseling for entire populations across wide geographic areas, thus providing broad access to tobacco intervention services. A final related goal is to target specific populations for interventions, such as pregnant women, older adults, adolescents, patients in health care systems, and ethnic minority groups to potentially reduce disparities in care. Both functions can enhance the public health reach of evidence based tobacco intervention. 
Range of Services. A variety of services can be offered through quitlines. Based on a survey of 29 US state quitlines, $\mathrm{Zhu}^{6}$ reported that at least $90 \%$ of quitlines offered mailed materials, referrals to other resources, and reactive counseling (smokers call in to speak with a counselor), 87\% offered proactive services (counselors call out to smokers on a scheduled basis), just under half (42-43\%) offered help obtaining nicotine replacement/bupropion (Zyban) and web chat services, and between one quarter and one third offered information on nicotine replacement/bupropion and taped messages. Most quitlines also offered at least some services for specific populations, with $90 \%$ providing services for pregnant smokers, nearly two thirds offering services for smokeless tobacco users, and about half (46-50\%) providing services for adolescent smokers and/or Medicare recipients.

Quitline Models. Several models of quitline services are in use. Quitline services may be embedded in broader hotline services. For example, the Cancer Information Service (CIS) hotline provides information and materials on cancer in general, as well as special materials and reactive counseling for smoking. More typical are smoking-specific quitlines. Within these quitlines, services may be offered using 1 of several models. Smokers who call may speak directly with a counselor. A somewhat different model has been used by our group in Rochester, NY, where callers hear a brief, taped daily message and then have the option of holding on to speak with a dispatcher who contacts an on-call counselor to return the call to the smoker within minutes. ${ }^{7} \mathrm{~A}$ third model is to present callers with a telephone tree for services, as in the Arizona state quitline, which offers choices including materials, messages, or counseling. Finally, the quitline may be answered by a screener in such states as California, and the screener either directly provides services such as mailing materials or arranges for proactive telecounseling callbacks. All of these models may offer materials as well as reactive and proactive telecounseling. No direct comparison of quitline models is available; thus, there is no evidence for greater effectiveness of any one model over others.

A range of models for counselor recruitment and training has also been implemented. Quitlines vary in the extent to which counselors have a background in counseling and related professional fields or are paraprofessional community or peer recruits. Counselors may be referred to as "quit coaches," "intervention specialists," or "counselors." Nonsmokers are typically used, although ex-smokers may be specifically recruited, as is the case for our Rochester quitline services. All counselors generally receive some type of training, although nationally accepted standards to identify and ensure training in core competencies have not yet been developed. Again, there is no evidence for the greater effectiveness of one counselor type compared with others.

\section{Evidence Base: Reactive Quitlines}

The Role of Promotion. Research has clearly demonstrated that broad community-based promotion increases quitline utilization. ${ }^{8-12}$ For example, Ossip-Klein et $\mathrm{al}^{8}$ demonstrated that free television, radio, and newspaper promotions tripled call rates compared with promotion through smoking cessation settings alone. Quitlines can be promoted through multiple channels, including direct media promotion, tagging the quitline number to media antismoking campaigns, health care providers, health systems, work sites, and schools. Opportunities for innovation exist. For example, some countries are phasing in the listing of their quitline number on cigarette packs. Overall, advertising, particularly when tagged to antismoking media campaigns, increases call rates and may stimulate calls from those who do not otherwise seek help. ${ }^{11-12}$

The challenge to quitlines is to identify and maintain a balance between promotion and utilization. It is critical to have the dollars to maintain a sufficiently high level of promotion to ensure adequate call rates that justify continuation of the quitline service, while not stimulating excessive demand that overwhelms the resources of the system. We conducted a nonrandom sampling of several of the established quitlines in North America to estimate utilization. Overall, 1.1 to $1.7 \%$ of adult smokers called a quitline over the course of a year. These rates are slightly lower than those reported for England $(4 \%)^{5}$ and Scotland (5.9\%), ${ }^{13}$ although all are in the low single-digit range. Similarly, Glasgow et $\mathrm{al}^{14}$ reported that $2.4 \%$ of HMO smokers used their quitline. Somewhat higher utilization rates have been found in some targeted populations. For example, we have found quitline utilization rates of 26 to $50 \%$ in our trials with both broad age range populations and mid-life and older adults, with 7 to $25 \%$ of the sample (25-50\% of callers) opting to speak with a quit coach. ${ }^{7,15}$

These data demonstrate that smokers will use quitlines if promoted on an ongoing basis. The relatively low utilization rates on a community level underscore the need to offer services across large population bases to ensure adequate numbers of calls and also suggest that we have not yet maximized the reach of quitlines. Targeted population studies and data from other countries point to the potential for increasing utilization with the concurrent need to ensure that adequate funding and resources are available to support higher call volumes.

Evidence Base. One community-based controlled trial of quitlines has been conducted by our group. ${ }^{7}$ Ten counties were randomized to quitline access or no quitline access, 1813 smokers were 
followed over 18 months, and all subjects were given a self-help manual. Self-reported abstinence was verified by significant other report, cotinine, and alveolar carbon monoxide levels. Overall, 35.9\% called the quitline at least once by 18 months, and $8.7 \%$ spoke with a counselor at least once. Across virtually all follow-up intervals and measures, abstinence rates were significantly higher in quitline counties relative to control counties, with odds ratios of 1.43 to 1.47 at 18 months. This suggests the effectiveness of the reactive quitline; the magnitude of the effect suggests that there may have also been a "ripple" effect for nonusers for whom knowledge of the presence of the quitline may have stimulated a quit attempt.

Several studies have examined whether reactive interventions are differentially effective. No differences have been reported among various counseling strategies (stage of change based, behavioral coping, cognitive coping, self-generated, informational). ${ }^{16,17}$ Similarly, no differences have been found among types of materials sent, ${ }^{18,19}$ although targeted materials may be of value in enhancing recruitment of specific populations. The results suggest that small differences in supportive- and/or coping skills-based counseling protocols and materials are unlikely to influence outcomes.

\section{Evidence Base: Proactive Telecounseling}

Evidence Base. Three meta-analyses support the efficacy of proactive telecounseling. ${ }^{1-3}$ For example, Lichtenstein et $\mathrm{al}^{1}$ conducted a meta-analysis of 13 studies of proactive calls and found an overall intervention effect at both short- and long-term followups (odds ratio, 1.20-1.34). In addition, the real-world effectiveness of proactive calls has recently been demonstrated using an innovative methodological approach to a natural experiment in California. ${ }^{20}$

Variability has been found in results for individual applications of proactive calls. Efficacy has been demonstrated when proactive telecounseling is used as the primary intervention ${ }^{21-25}$ and to augment hospital initiated interventions for cardiac patients ${ }^{26,27}$ or general hospitalized patients. ${ }^{28,29}$ There seems to be a dose response effect, with multiple calls producing higher abstinence relative to a single call. ${ }^{22}$ Promising results have been found for a small number of studies using telephone counseling to extend effects of a school-based prevention program,$^{30}$ to promote requitting among smokers with recent unsuccessful quit attempts, ${ }^{31}$ to support short-term abstinence among low-income women provided with free nicotine patches and proactive calls, ${ }^{32}$ to promote short-term abstinence among smokeless tobacco users, ${ }^{33}$ and as part of a tailored intervention for African American smokers. ${ }^{34}$

Mixed results have been found for proactive calls to augment personalized written feedback, with a 12-month effect found for calls when combined with stage-tailored materials and expert system personalized feedback in the second of 2 trials by Prochaska et $\mathrm{al}^{35,36}$ and higher short-term abstinence reported in a trial by Curry et al. ${ }^{37}$ Mixed results have also been found for proactive calls to support nicotine replacement use. No effect was reported by earlier trials, ${ }^{38-40}$ although promising results have been found in a more recent trial by Solomon and colleagues ${ }^{32}$ and in preliminary data from our trials. There is some evidence that the availability of proactive telecounseling may be viewed positively by physicians and health systems, who may be more likely to intervene with patients in the context of this referral source. ${ }^{41,42}$

No effect has yet been demonstrated for proactive calls as a follow-up for smokers in alcohol treatment ${ }^{43}$ or smoking clinics, ${ }^{31}$ although the latter case may indicate a difference in populations served. For example, a recent report found that when given the choice between clinics or proactive calls, two thirds chose the calls as an alternative to clinics. ${ }^{44}$ Data are not yet available for the efficacy of internet/web site/web chat interventions, or for interactive voice recording interventions. Data are also not yet available on the timing of calls, although some investigators clinically suggest timing calls around the quit date and during periods of greatest relapse risk.

\section{Special Application: Adolescents}

Adolescents represent a key population for smoking cessation intervention. Estimates are that 2145 adolescents begin smoking each day in the United States. ${ }^{45}$ Although there has been considerable research on smoking prevention among youth, little data are currently available on smoking intervention for this population. At least 4 projects are currently underway examining telecounseling for adolescent tobacco use. Zhu and colleagues are testing proactive telecounseling for adolescents through the California state quitline, Brown and colleagues are testing a multicomponent intervention including telecounseling for youth with comorbid psychopathology, Lando and colleagues are testing telephone follow-ups after a dental intervention, and our group is examining teen web site use, including telecounseling-type services delivered through instant messaging chat using the Monroe County GottaQuit program. Although outcome data are not yet available, early utilization data suggest that 0.6 to $2.0 \%$ of adolescents are using quitlines or web chats, which is similar to usage observed among adult smokers for community quitlines.

\section{Future Directions}

Quitlines provide an infrastructure for the translation of evidence-based interventions into public health applications. A "critical mass" of quitlines 
has been achieved through state, health system, and national efforts, and the field is continuing to grow both nationally and globally. The field is sufficiently established that a number of groups nationally are critically examining how to best move the agenda forward to maximize the reach and effectiveness of quitlines and to identify and address gaps. To this end, a National Conference of Smoking Cessation Quitlines was held in Phoenix in May 2002. This conference was funded by national agencies/foundations, including the National Cancer Institute, Centers for Disease Control and Prevention, Robert Wood Johnson Foundation, American Cancer Society, American Legacy Foundation, Health Canada, and National Cancer Institute of Canada. The goal of the conference was to bring together quitline providers, researchers, and funders to develop a summary of issues and recommendations for research and service needs. The consensus of the group was that the field has demonstrated that quitlines can work; the next step is to ask what is best under what circumstances and how can we refine and improve our operations. A series of recommendations was developed after a series of invited addresses, plenary groups, and working group sessions. These recommendations will be published in detail separately. Continuing planning, ongoing research, and a focus on translating research findings to quitline applications can maximize the public health reach and impact of this important intervention modality.

\section{References}

1. Lichtenstein E, Glasgow RE, Lando HA, et al. Telephone counseling for smoking cessation: rationales and review of evidence. Health Ed Res 1996;11:243-57.

2. Fiore MC, Bailey WC, Cohen SJ, et al. Treating tobacco use and dependence. Clinical practice guideline. Rockville (MD): US Department of Health and Human Services, Public Health Service; 2000.

3. Stead LF, Lancaster T. Telephone counseling for smoking cessation. Cochrane Database Syst Rev 2001;(2):CD002850.

4. Best practices for comprehensive tobacco control programsAugust 1999. Atlanta: US Department of Health and Human Services, Centers for Disease Control and Prevention, National Center for Chronic Disease Prevention and Health Promotion, Office on Smoking and Health; 1999.

5. Owen L. Impact of a telephone helpline for smokers who called during a mass media campaign. Tob Control 2000;9: $148-54$.

6. Zhu S-H. A survey of quitlines in North America. Paper presented at the North American Conference of Smoking Cessation Quitlines; 2002 May 8-10; Phoenix, Arizona.

7. Ossip-Klein DJ, Giovino GA, Megahed N, et al. Effects of a smokers' hotline: results of a 10-county self-help trial. J Consult Clin Psychol 1991;59:325-32.

8. Ossip-Klein DJ, Shapiro RM, Stiggins J. Freedom Line: increasing utilization of a telephone support service for exsmokers. Addict Behav 1984;9:227-30.

9. Pierce JP, Anderson DM, Romano RM, et al. Promoting smoking cessation in the United States: effect of public ser- vice announcements on the Cancer Information Service telephone line. J Natl Cancer Inst 1992;84:677-83.

10. Cummings KM, Sciandra R, Davis S, et al. Results of an antismoking media campaign utilizing the Cancer Information Service. J Natl Cancer Inst Monogr 1993;(14):113-8

11. Zhu S-H, Anderson CM, Johnson CE, et al. A centralised telephone service for tobacco cessation: the California experience. Tob Control 2000;9(Suppl 2):II48-55.

12. Wakefield M, Borland R. Saved by the bell: the role of telephone helpline services in the context of mass-media anti-smoking campaigns. Tob Control 2000;9:117-9.

13. Platt S, Tannahill A, Watson J, et al. Effectiveness of antismoking telephone helpline: follow up survey. BMJ 1997; 314:1371-5.

14. Glasgow RE, Lando H, Hollis J, et al. A stop-smoking telephone help line that nobody called. Am J Public Health 1993;83:252-3.

15. Ossip-Klein DJ, Krusch D, Carosella AM. Self-help interventions for older smokers. Tob Control 1997;6:188-93.

16. Thompson B, Kinne S, Lewis FM, et al. Randomized telephone smoking-intervention trial initially directed at blue-collar workers. J Natl Cancer Inst Monogr 1993;14:10512.

17. Shapiro RM, Ossip-Klein DJ, Stiggins J. Freedom Line Phase II: the evaluation of a relapse-prevention hotline for ex-smokers. Paper presented at the 17th Annual Convention of the Association for Advancement of Behavior Therapy/ World Congress on Behavior Therapy; 1983 Dec; Washington DC.

18. Davis SW, Cummings KM, Rimer BK, et al. The impact of tailored self-help smoking cessation guides on young mothers. Health Educ Q 1992;19:495-504.

19. Balanda KP, Lowe JB, O'Connor-Fleming ML. Comparison of two self-help smoking cessation booklets. Tob Control 1999;8:57-61.

20. Zhu S-H, Anderson CM, Tedeschi GJ, et al. Evidence of real-world effectiveness of a telephone quitline for smokers. N Engl J Med 2002;347:1087-93.

21. Orleans CT, Schoenbach VJ, Wagner EH, et al. Self-help quit smoking interventions: effects of self-help materials, social support instructions, and telephone counseling. J Consult Clin Psychol 1991;59:439-48.

22. Zhu S-H, Stretch V, Balabanis M, et al. Telephone counseling for smoking cessation: effects of single session and multiple session interventions. J Consult Clin Psychol 1996; 64:202-11.

23. Lando HA, Hellerstedt WL, Pirie PL, et al. Brief supportive telephone outreach as a recruitment and intervention strategy for smoking cessation. Am J Pub Health 1992;82: $41-6$.

24. Curry SJ, McBride C, Grothaus LC, et al. A randomized trial of self-help materials, personalized feedback, and telephone counseling with nonvolunteer smokers. J Consult Clin Psychol 1995;63:1005-14.

25. Rimer BK, Orleans CT, Fleisher L, et al. Does tailoring matter? The impact of a tailored guide on ratings and shortterm smoking-related outcomes for older smokers. Health Educ Res 1994;9:69-84.

26. Taylor CB, Houston-Miller N, Killen JD, et al. Smoking cessation after acute myocardial infarction: effects of a nursemanaged intervention. Ann Intern Med 1990;113:118-23.

27. DeBusk RF, Houston-Miller N, Superko HR, et al. A case-management system for coronary risk factor modification after acute myocardial infarction. Ann Intern Med 1994; 120:721-9. 
28. Miller NH, Smith PM, DeBusk RF, et al. Smoking cessation in hospitalized patients. Results of a randomized trial. Arch Intern Med 1997;24:409-15.

29. Stevens VJ, Glasgow RE, Hollis JF, et al. A smokingcessation intervention for hospital patients. Med Care 1993; 31:65-72

30. Elder JP, Wildey M, de Moor C. et al. The long-term prevention of tobacco use among junior high school students: Classroom and telephone interventions. Am J Public Health 1993;83:1239-44.

31. Lando HA, Pirie PL, Roski J, et al. Promoting abstinence among relapsed chronic smokers: the effect of telephone support. Am J Public Health 1996;86:1786-90.

32. Solomon LJ, Scharoun GM, Flynn BS, et al. Free nicotine patches plus proactive telephone peer support to help lowincome women stop smoking. Prev Med 2000;31:68-74.

33. Severson HH, Andrews JA, Lichtenstein E, et al. A self-help cessation program for smokeless tobacco users: comparison of two interventions. Nicotine Tob Res 2000;2:36370.

34. Orleans CT, Boyd NR, Bingler R, et al. A self-help intervention for African American smokers: tailoring Cancer Information Service counseling for a special population. Prev Med 1998;27:S61-70.

35. Prochaska JO, DiClemente CC, Velicer WF, et al. Standardized, individualized, interactive, and personalized selfhelp programs for smoking cessation. Health Psychol 1993; 12:399-405.

36. Prochaska JO, Velicer WF, Fava JL, et al. Counselor and stimulus control enhancements of a stage-matched expert system intervention for smokers in a managed care setting. Prev Med 2001;32:23-32.
37. Curry SJ, McBride C, Grothaus LC, et al. A randomized trial of self-help materials, personalized feedback, and telephone counseling with nonvolunteer smokers. J Consult Clin Psychol 1995;63:1005-14.

38. Lando HA, Rolnick S, Klevan D, et al. Telephone support as an adjunct to transdermal nicotine in smoking cessation. Am J Public Health 1997;87:1670-4.

39. Ockene JK, Kristeller J, Goldberg R, et al. Increasing the efficacy of physician-delivered smoking interventions: a randomized trial. J Gen Intern Med 1991;6:1-8.

40. Reid RD, Pipe A, Dafoe WA. Is telephone counseling a useful addition to physician advice and nicotine replacement therapy in helping patients to stop smoking? A randomized controlled trial. Can Med Assoc J 1999;160:1577-81.

41. Zhu S-H, Tedeschi G, Anderson CM, et al. Telephone counseling as adjuvant treatment for nicotine replacement therapy in a "real-world" setting. Prev Med 2000;31:357-63.

42. Marcy TW, Solomon LJ, Dana GS, et al. A smoking cessation telephone resource: feasibility and preliminary evidence on the effect on health care provider adherence to smoking cessation guidelines. Tob Control 2002;11:84.

43. Leed-Kelly A, Russell KS, Bobo JK, et al. Feasibility of smoking cessation counseling by phone with alcohol treatment center graduates. J Subst Abuse Treat 1996;13:203-10.

44. McAfee T, Sofian NS, Wilson J, et al. The role of tobacco intervention in population-based health care: a case study. Am J Prev Med 1998;14:46-52.

45. Summary of findings from the 2000 National Household Survey on Drug Abuse. NHSDA series H-13, DHHS publication no. (SMA) 01-3549. Rockville (MD): Office of Applied Studies, Substance Abuse and Mental Health Services Administration; 2001. 